\title{
Learning to make a difference: facilitating improvements in care by enabling core medical trainees to develop skills in quality improvement
}

\author{
Authors: Emma Vaux ${ }^{\mathrm{AB}}$ and Katharine Woodall ${ }^{\mathrm{A}}$
}

\section{Aims}

To support the learning and development of new and relevant skills in quality improvement (QI) methodology by junior doctors to enable them to deliver effective QI projects at the frontline.

\section{Methods}

Learning to Make a Difference (LTMD) began as a pilot project in 2010. It is now established as part of core medical training. Trainees are asked to identify a topic for improvement and to complete their project within their training year. Each trainee is supported by a consultant supervisor, LTMD practical toolkit and the LTMD website, as well as the central implementation team. In addition, six consultants from across the UK, experts in QI, provide further support at regional level. Innovatively, they also run weekly WebEx sessions for trainees to dial into for help and support and to share learning.

\section{Results}

The successful LTMD pilot demonstrated more evidence of benefit to patients than any of the current workplace-based assessments. Quality Improvement Impact Assessment Tools (QIPATs) from 2013/14 have substantiated this, showing many innovative and diverse projects that have produced significant patient gains, with over $55 \%$ of projects being judged as achieving an exemplary standard.

The curriculum has been updated. There is now a change to the requirement for a core medical trainee 'to undertake an audit', so the trainee may do a quality improvement project or complete an audit cycle using QI methodology, in a training year.

\section{Conclusions}

Having created capability and capacity for trainees to be able to ask the question 'what can I do to make a difference' without having to wait to be asked to improve, we have empowered trainees to resist the theme of 'learned helplessness' and to have a means to respond to the messages from the Francis and Berwick reports and to make a positive difference to patient care. There has been agreement from the Heads of School of Medicine Committee and RCP specialty advisory committees to spreading the success of the LTMD approach to all doctors in training in all the medical specialties. The project has also been leading the way for cross-college collaboration, working with the Academy of Medical Royal Colleges (AoMRC) Specialist Training Committee, to establish an AoMRC Task and Finish group. This group will enhance cross-college collaboration on QI, share learning, expertise, resources (eg training for supervisors) and support, as QI becomes part of trainees' everyday experience.

\section{Conflict of interest statement}

None. 